\title{
IONOSPHERE STUDIES DURING PARTIAL SOLAR ECLIPSE OF FEBRUARY 3, 1935
}

\author{
By Samuel S. Kirby, Theodore R. Gilliland, and Elbert B. Judson
}

\section{ABSTRACT}

Virtual height and critical-frequency measurements of the several regions of the ionosphere were made during the day of the solar eclipse of February 3, 1935, and during several days before and after the eclipse day. The eclipse was found to produce a decrease of the critical frequency of each region. The decrease of critical frequency was approximately in time phase with the eclipse, thus indicating an ionizing agency (probably ultraviolet light), originating in the sun and propagated at approximately the velocity of light. The decrease of equivalent electron density in each region during the eclipse was compared with the decrease in the exposed area of the sun's disk, and found to indicate that the ionization of the normal E region was diminished when the ionizing agency was decreased, by recombination of + and - charges, while the ionization of the $\mathrm{F}_{2}$ region and a high stratum of the $\mathrm{E}$ was diminished by a process of attachment of electrons to neutral particles.

\section{CONTENTS}

I. Introduction

Page

II. Experimental data

III. Discussion of data

IV. Conclusions

V. References

\section{INTRODUCTION}

Previous to the solar eclipse of August 31, 1932, no quantitative determinations of the ionization densities of the several regions of the ionosphere had been made at the time of an eclipse. Measurements made by the National Bureau of Standards [1] ${ }^{1}$ and other North American observers [2,3] during this eclipse indicated that the main source of ionization of the $\mathrm{E}$ and $\mathrm{F}_{1}$ regions was ultraviolet light. Similar effects for the $\mathrm{F}_{2}$ region were not definitely found. Rose [4] reported a 30-percent decrease in ionization density but was not sure that he had followed the $\mathrm{F}_{2}$ critical frequency all of the time. The National Bureau of Standards found a small decrease of ionization density during this eclipse, but the effect found was of approximately the same magnitude as variations found on some of the control days. The observations of Kenrick and Pickard [5] and Mimno and Wang [6] seem to apply to the $\mathrm{F}_{1}$ region instead of the $\mathrm{F}_{2}$.

The 1932 eclipse was total through the Province of Ontario, Canada, and Northeastern United States, where it could easily be observed.

1 The figures given in brackets throughout the text correspond to the numbered references at the end of this paper. 
At Washington, D. C., about 90 percent of the area of the sun's disk was obscured. The eclipse occurred during early and midafternoon. The season, hour of day, and percentage of totality seemed to be favorable for definite results. Such results were obtained for the E and $\mathrm{F}_{1}$ regions but not for the $\mathrm{F}_{2}$.

The partial solar eclipse of February 3, 1935, offered an opportunity for further study of the effects of eclipses on the ionosphere. The hour of day (late forenoon) seemed favorable, but the season and percentage of totality (35 percent of area of sun obscured) did not seem favorable for good results.

The effect of a winter eclipse on the $\mathrm{F}_{2}$ region was of especial interest. It had been observed for several years by the National Bureau of Standards [7, 8] at Washington and the Bell Telephone Laboratories [9] at Deal, N. J., that the ionization density of the $\mathrm{F}_{2}$ region was not a simple function of the intensity of solar radiation. The ionization densities reached maxima at about sunset in summer and shortly after noon in winter, the winter day values being higher than the summer day values [10]. The ionization density followed nearly in phase with the intensity of received solar radiation during the winter day. Therefore there was a possibility that the effect of an eclipse in the $\mathrm{F}_{2}$ region would be more clearly indicated in the winter than in the summer.

The technique and equipment available for the observations in 1935 were considerably better than those available in 1932 . The multifrequency automatic recorder [11] was available to measure the virtual heights and critical frequencies for the band 2,500 to 4,400 $\mathrm{kc} / \mathrm{s}$ with a continuous frequency variation. This frequency band was swept through 10 times per hour on the eclipse day and also the 2 days before and the 2 days after. Each sweep through this band required 4.75 minutes. The records obtained gave the critical frequencies for the $\mathrm{E}$ and $\mathrm{F}_{1}$ regions and during the eclipse three points for the $F_{2}$ region. The virtual heights and critical frequencies for the frequency band from $4,500 \mathrm{kc} / \mathrm{s}$ up to the $\mathrm{F}_{2}$ critical frequencies were determined by manually operated equipment with which the frequency was varied in steps, usually $200 \mathrm{kc} / \mathrm{s}$. Some manual observations were also made at frequencies below $2,500 \mathrm{kc} / \mathrm{s}$. The general method of observation was to increase the frequency of the pulse transmitter from low values to high and measure the virtual heights until the critical frequency was exceeded. This general procedure has been followed at the National Bureau of Standards since August 1930 [12], the technique improving with experience and improvements of equipment. The ionization densities were computed from critical frequencies by equation 1 given later.

\section{EXPERIMENTAL DATA}

The data include critical-frequency and virtual-height measurements of the several ionized regions of the ionosphere, made by the methods just described. Figure 1 shows the photographic records obtained on the frequency band 2,500 to $4,400 \mathrm{kc} / \mathrm{s}$. Scales for virtual heights and frequencies are indicated in the lower left-hand corner of figure 1. Critical frequencies are indicated by a more or less sharp rise of virtual height or a point of inflection. The critical 


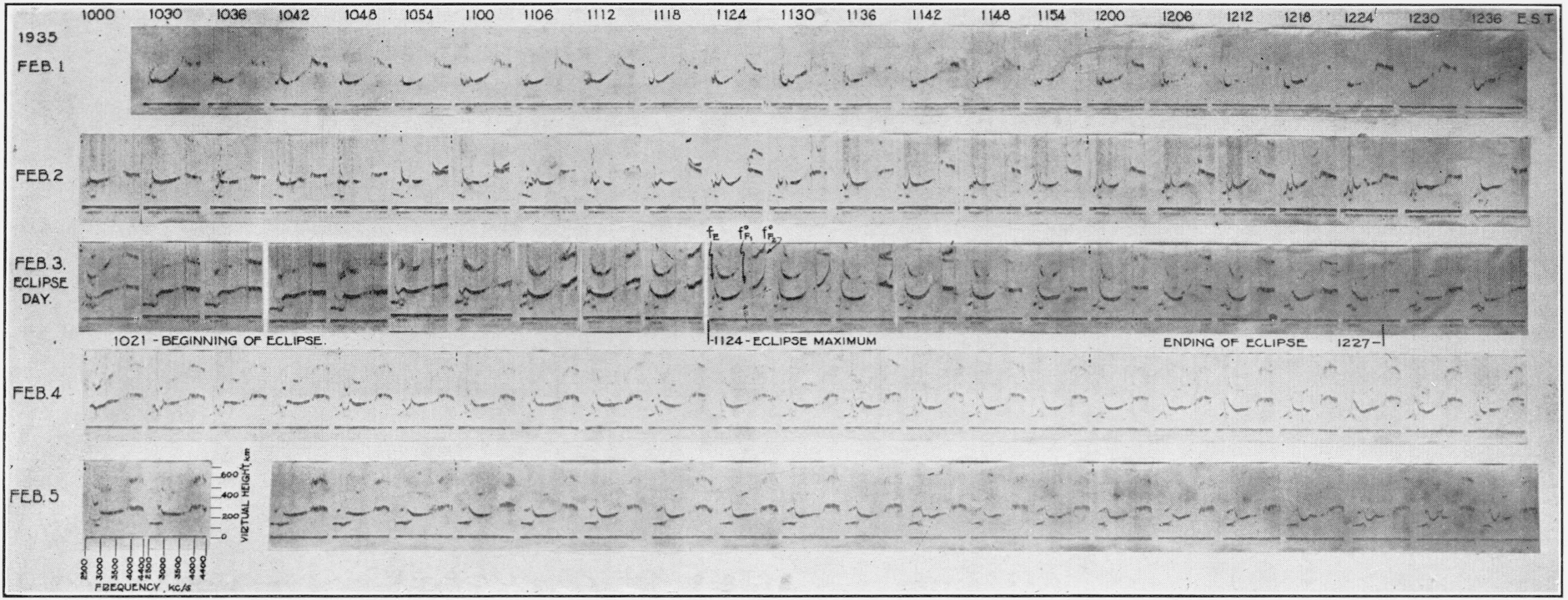

FIGURE 1.-Automatic multifrequency records of ionosphere heights and critical frequencies for day of eclipse and 2 days before and 2 days after eclipse. 
frequency ${ }^{2}$ of a given ionized region is the lowest frequency which will penetrate that region. The $f_{\mathrm{E}}$ and $f_{\mathrm{F} 1}^{\mathrm{O}}$ are indicated on these records. In addition to the manual measurements, three points of $f^{\circ}{ }^{\circ}$ were obtained with the automatic recorder near the maximum of the eclipse.

The $\mathrm{E}$ region was complex throughout this series of measurements. It appeared to be stratified. Two or three critical frequencies appeared much of the time. The values of these critical frequencies varied independently, and frequently one critical frequency would disappear or a new one appear between sweeps of six-minutes separation. The two criticals appearing on February 3, between 1020 and 1112 EST, (fig. 4), are examples of this complexity. It was often impossible to follow a particular $\mathrm{E}$ critical frequency through a series of records taken during successive six-minute periods. Some experimenters [9] have distinguished the several stratifications below the $\mathrm{F}_{1}$ region by separate names. With the conditions existing during the period of these experiments it did not appear that this could be done consistently. For the reasons given above the multiple $\mathrm{E}$ critical frequencies have been averaged for the four control days.

The critical frequencies of the $\mathrm{F}_{1}$ region were single but not sharply defined. The poor definition is characteristic of winter $f_{\mathrm{F} 1}$. No double refraction was observed.

Three of the photographic records taken during the eclipse indicate $f_{\mathrm{F}_{2}}^{o}$. On the first of these, the record beginning at 1124 EST, $f_{\mathrm{F}_{2}}^{\circ}$ is indicated at the end of the record $4,400 \mathrm{kc} / \mathrm{s}$. On the record beginning at $1130 \mathrm{EST}, f_{{ }_{F_{2}}}^{\circ}$ is indicated clearly at $4,330 \mathrm{kc} / \mathrm{s}$. The time at which this critical frequency was recorded was approximately 1134.5 EST. On the record beginning at 1136 EST $f_{\mathrm{r}_{2}}^{\circ}$ was again indicated at $4,400 \mathrm{kc} / \mathrm{s}$. These three records indicate the time of minimum $f_{\mathrm{F}_{2}}^{\circ}$ positively to within 6 minutes and by interpolation probably to the nearest minute.

In figure 2 the data are shown for $f_{\mathrm{E}}$ and $f_{\mathrm{F}_{1}}{ }$ for February 1, 2, 4, and 5 obtained from the records of figure 1 . No attempt was made in this figure to distinguish critical frequencies for any individual day.

In figure 3 are shown the data for $f_{{ }_{\mathbf{F}_{2}}}^{\circ}$ for the eclipse day and several days before and after the eclipse. The data for each day are shown by a separate graph in this figure. It will be noted that there was considerable variation of critical frequency from day to day. This makes it somewhat difficult to compare the results of any given day on which a special phenomenon such as an eclipse occurs with the average of several normal days, because it is not known for certain what the results would have been on the given day had not the special phenomenon occurred. The Cheltenham Magnetic Observatory of the United States Coast and Geodetic Survey reported moderate magnetic disturbances for February 1 and 2. The magnetograms indicate that the disturbance of February 2 was much less severe than that on Feb. 1. The $\mathrm{F}_{2}$ critical frequencies were much lower on these two days than on the other control days. Some other data obtained at the National Bureau of Standards indicate that this correspondence between magnetic disturbances and low $f_{\mathbf{F}_{2}}$ frequently exists.

${ }^{2} f_{\mathbf{w}}=$ critical frequency for the $\mathrm{E}$ region; $f^{\circ} \mathbf{v}_{\mathbf{1}}=$ critical frequency for the $\mathrm{F}_{1}$ region, ordinary ray; $f x_{x_{1}}=$ critical frequency for the $F_{1}$ region, extraordinary ray; $f o_{\mathbf{x}_{2}}=$ critical frequency for the $F_{2}$ region, ordinary ray; $f_{\mathbf{x}_{2}}=$ critical frequency for the $\mathrm{F}_{2}$ region, extraordinary ray. 


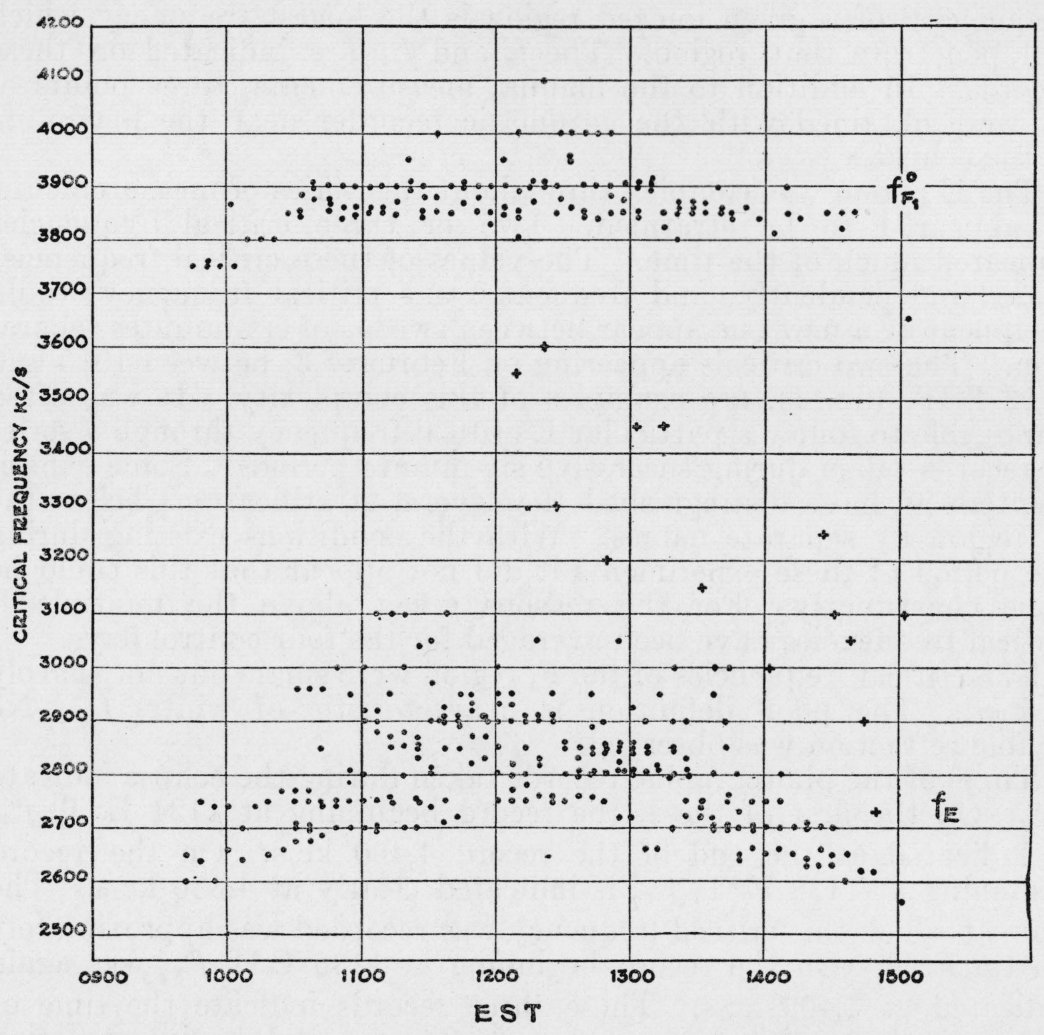

$f_{F_{1}}^{\circ}$ AND $f_{E}$ FOR FEBRUARY $1,2,4$ AND 5,1935

Figure 2.-Critical frequency data for control days for $\mathrm{E}$ and $\mathrm{F}_{1}$ regions.

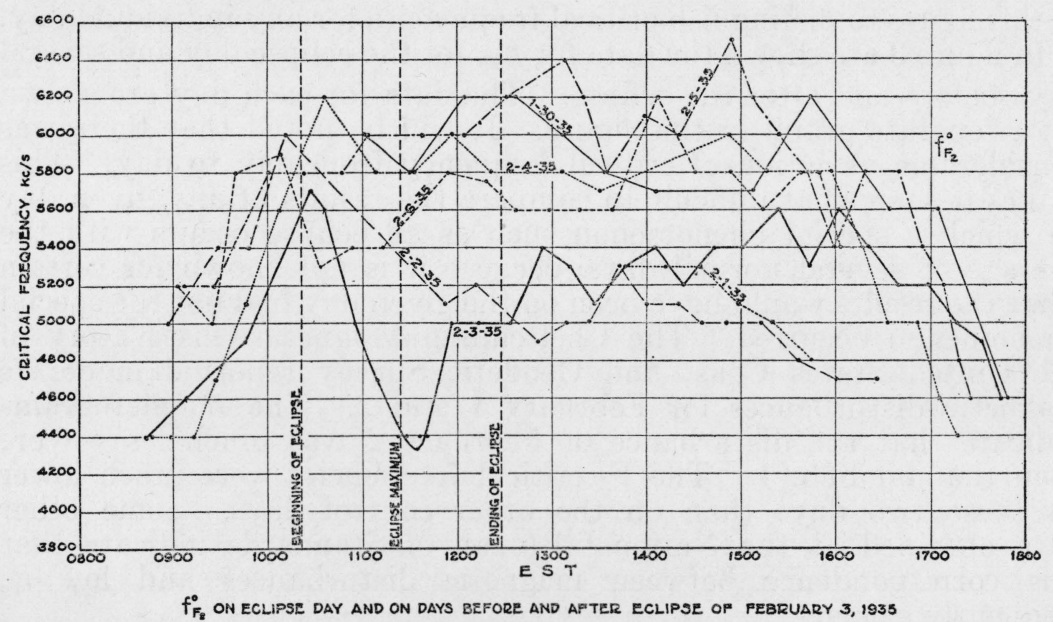

Figure 3.-Critical frequency data for eclipse day and control days for $\mathrm{F}_{2}$ region. 
Figures 1, 2, and 3 present the experimental data obtained in this investigation.

\section{DISCUSSION OF DATA}

Since there was a complexity of critical frequencies in the $\mathrm{E}$ region, and the critical values for a given region could not be traced through with certainty, an average was made of all the points obtained except a few widely scattered points indicated by + in figure 2 .

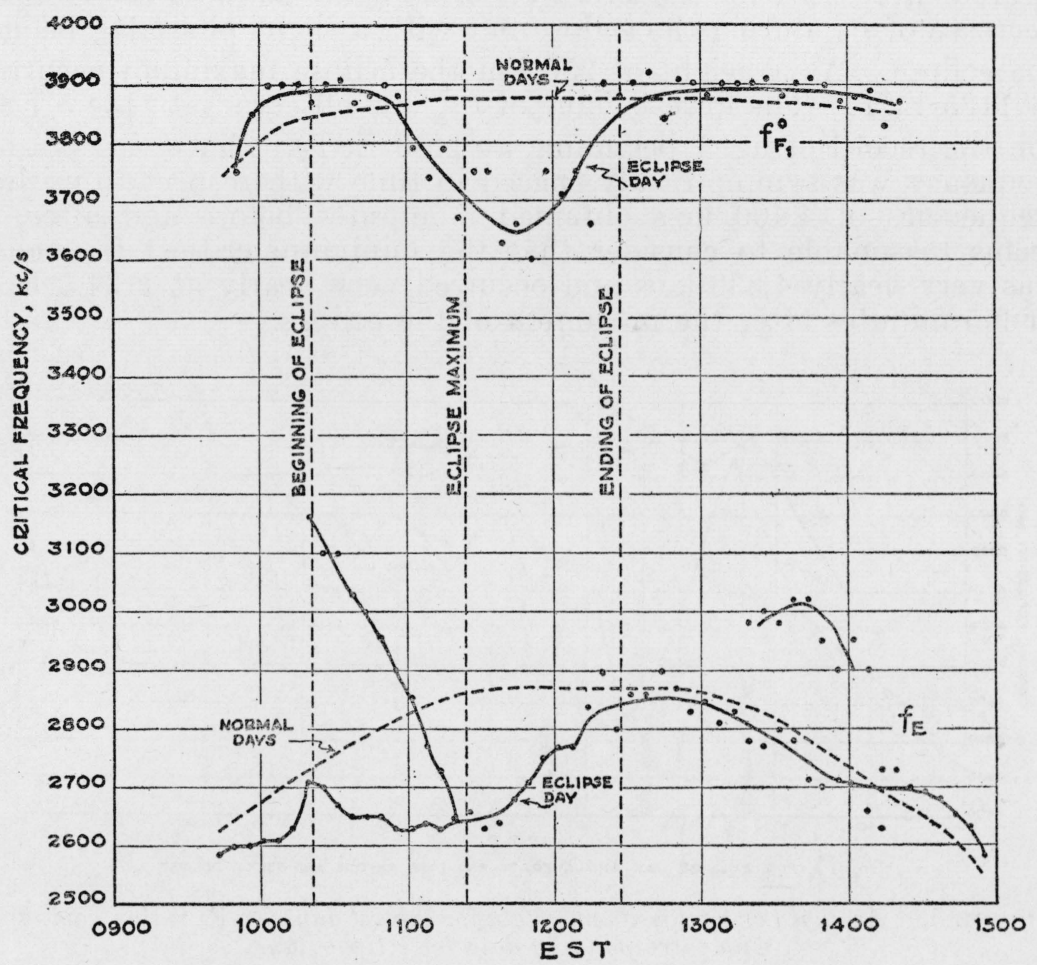

$f_{E}$ AND $f_{F_{1}}^{\circ}$,FOR ECLIPSE DAY AND FOR DAYS BEFORE AND AFTER ECLIPSE

Figure 4.-Averaged critical frequency data for control days for $\mathrm{E}$ and $\mathrm{F}_{1}$ regions compared with corresponding data for eclipse day.

These points were not used in the average simply because they were so widely scattered that they did not seem to belong to the E region. This average, and the individual critical frequencies obtained on the eclipse day, are shown in the lower part of figure 4 . The branch beginning with a critical frequency of $3,160 \mathrm{kc} / \mathrm{s}$ at about $1020 \mathrm{EST}$ and ending about 1120 EST by combining with the more normal appearing $f_{\mathrm{E}}$ graph is of special interest although its significance is not clear. The critical frequencies forming this branch can be seen clearly in figure 1. Except for this branch the $f_{\mathrm{z}}$ was less complex on 
the eclipse day than on the control days. This figure indicates a decrease of $f_{\mathrm{E}}$ during the eclipse.

The upper part of figure 4 shows $f_{F_{1}}$ for the eclipse day and the average for the control days. This figure indicates a decrease of $f_{\mathbf{F}_{1}}^{\circ}$ during the eclipse.

The $f_{{ }_{F_{2}}}^{\circ}$ for the eclipse day and the average for the control days is shown in figure 5. All of the data for the control days shown in figure 3 were used for the average. This figure shows a pronounced decrease of $f_{{ }_{r_{2}}}^{\circ}$ during the eclipse but with a slight phase lag behind the eclipse. At a height of $250 \mathrm{~km}$ the eclipse maximum occurred at 1125 EST. The lowest value of $f_{F_{2}}$ was observed at 1134.5 EST (on the record of fig. 1 beginning at 1130 EST). Since this critical frequency was symmetrically spaced in time with respect to critical frequencies of $4,400 \mathrm{kc} / \mathrm{s}$ obtained 6 minutes before and after, it seems reasonable to consider that the minimum critical frequency was very nearly $4,330 \mathrm{kc} / \mathrm{s}$ and occurred very nearly at $1134.5 \mathrm{EST}$ or 9.5 minutes after the maximum of the eclipse.

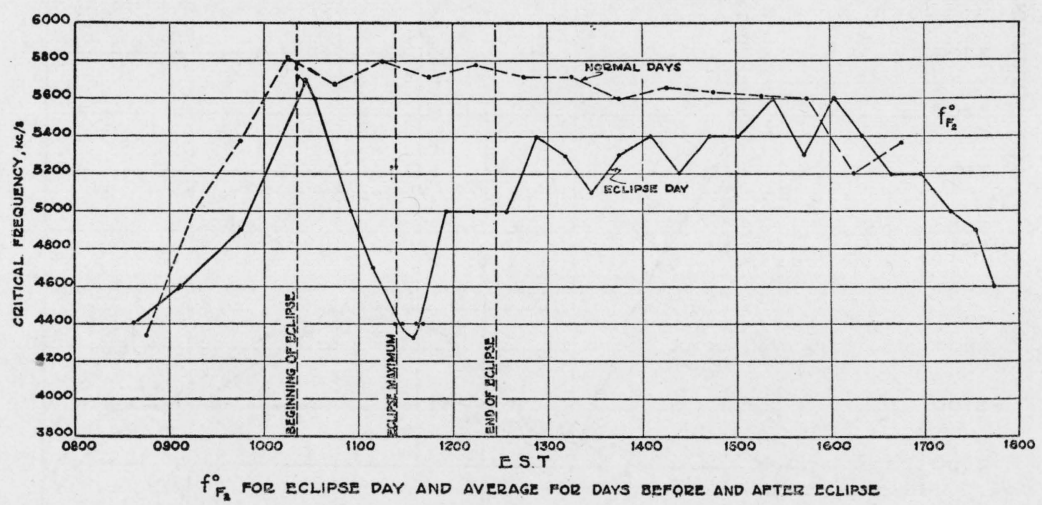

FIGURE 5.-Averaged critical frequency data for control days for $\mathrm{F}_{2}$ region compared with corresponding data for eclipse day.

Immediately before the eclipse, $f_{{ }^{2} 2}$ was nearly normal, while after the eclipse maximum it rose rapidly at first but later ceased to rise rapidly but reached normal between 1500 to 1600 EST. This effect might conceivably have been caused by the eclipse, but it is more likely that the normal value of $f_{F_{2}}^{\circ}$ was low during the eclipse day as on February 1 and 2. During the night following the eclipse $f_{\mathrm{F} 2}^{\mathrm{x}}$ remained below $2,500 \mathrm{kc} / \mathrm{s}$ from the hour $2030 \mathrm{EST}$ to sunrise. This represents an exceptionally long sustained low value of ionization density of the $\mathrm{F}_{2}$ region, but it has been recorded on other nights. It is not likely that this effect was caused by the eclipse. The ionization density during the night preceding the eclipse was also very low.

In the following paragraphs, let $N_{1}$ equal the ion density on the normal or control days, $N_{2}$ the ion density on the eclipse day, and $f_{1}^{\circ}$ and $f^{1}{ }_{2}$ the critical frequencies of the ordinary ray on the control days and eclipse day, respectively. The ratios $\frac{N_{2}}{N_{1}}$ and $\left(\frac{N_{2}}{N_{1}}\right)^{2}$ will be com- 
pared with the percentage of the sun's disk which was exposed on the eclipse day.

From familiar theory we may write the following equation expressing the relation between critical frequency and equivalent electron density:

$$
N=1.24\left(f^{\circ}\right)^{2} \cdot 10^{-2},
$$

where $N=$ equivalent electrons per $\mathrm{cm}^{3}$ for a given region, and $f^{\circ}=$ the critical frequency in $\mathrm{kc} / \mathrm{s}$ for the ordinary ray for the same region. For heavy ions equation 1 would have the same form but a greater numerical constant. Equation 1 is well known and is obtained directly from the fundamental theory of Eccles [13] and Larmor [14].

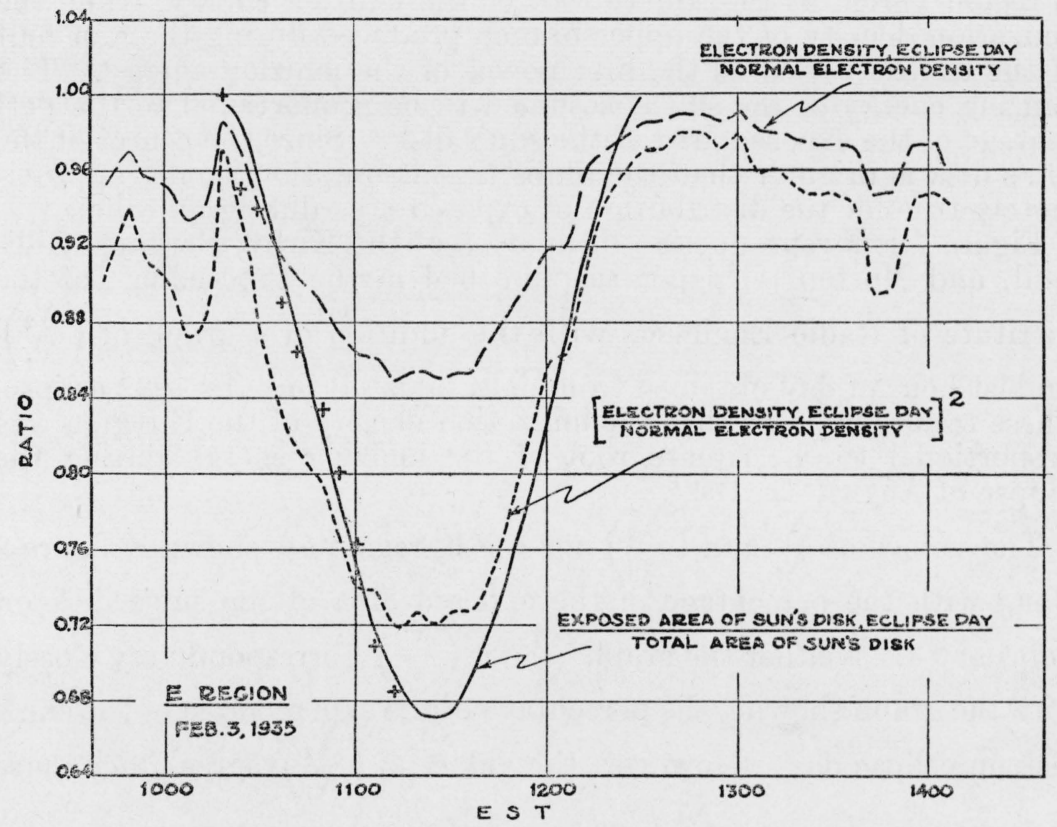

FIgURE 6.-Comparison of normal electron density of E region during 1935 eclipse, with exposed area of sun's disk

The + marks $=\frac{\text { electron density eclipse day }}{\text { normal electron density }}$ for upper branch of $\mathrm{E}$ region shown in figure 4.

From equation 1 we obtain

$$
\frac{N_{2}}{N_{1}}=\left(\frac{f_{2}^{0}}{f^{0}{ }_{1}}\right)^{2}
$$

The values of $\frac{N_{2}}{N_{1}}$ and $\left(\frac{N_{2}}{N_{1}}\right)^{2}$ for the E region are shown in figure 6 . The values of $f^{\circ}{ }_{1}$ and $f^{\circ}{ }_{2}$ were taken for each 6 -minute period from figures 4 and 5 , so that allowance has been made for the varying zenith angle of the sun. The ratios of the exposed area of the sun's disk on the eclipse day to the total area of the sun's disk are shown in 
the same figure. It may be seen that the graph for $\left(\frac{N_{2}}{N_{1}}\right)^{2}$ for the main $\mathrm{E}$ region corresponds closely to the graph showing the percentage of the exposed area of the sun's disk. The + marks in this figure represent values of $\frac{N_{2}}{N_{1}}$ instead of $\left(\frac{N_{2}}{N_{1}}\right)^{2}$ for the upper branch of the $\mathrm{E}$ region shown in figure 4. These values follow closely the graph showing the percentage of exposed area of the sun's disk. The value measured just before the eclipse began was assumed to be normal and was used for $N_{1}$. From the results shown in figure 6 it would appear that there were two different laws of recombination for these two strata of the $\mathrm{E}$ region. The ionization density for the lower $\mathrm{E}$ region varied as the square root of the ionizing energy, while the ionization density of the upper branch produced during the first half of the eclipse varied as the first power of the ionizing energy. The ionizing energy of the sun is assumed to be proportional to the percentage of the exposed area of the sun's disk. Since the center of the sun's disk is brighter than the limbs this assumption is only approximately true for the distribution of exposed area during an eclipse.

Figure 7 is a reproduction of figure 3 of the Kirby, Berkner, Gilliland, and Norton [1] paper as published in the Proceedings of the Institute of Radio Engineers with the addition of a graph of $\left(\frac{N_{2}}{N_{1}}\right)^{2}$ against hour of day obtained from data taken during the 1932 eclipse. These results indicate that the ionization density of the $\mathrm{E}$ region was proportional to the square root of the ionizing energy during the eclipse of August 31, 1932.

The values of $\frac{N_{2}}{N_{1}}$ and $\left(\frac{N_{2}}{N_{1}}\right)^{2}$ for the $\mathrm{F}_{1}$ region are shown in figure 8 along with the percentage of the exposed area of the sun's disk on February 3. Neither the graph $\frac{N_{2}}{N_{1}}$ nor $\left(\frac{N_{2}}{N_{1}}\right)^{2}$ correspond very closely with the graph showing the percentage of the exposed area of the sun's disk on eclipse day. However, the values of $\left(\frac{N_{2}}{N_{1}}\right)^{2}$ agree much more closely than those for $\frac{N_{2}}{N_{1}}$. These results indicate that it was more probable that the ion density of the $F_{1}$ region was proportional to the square root rather than the first power of the ionizing energy.

The values of $\frac{N_{2}}{N_{1}}$ and $\left(\frac{N_{2}}{N_{1}}\right)^{2}$ for the $\mathrm{F}_{2}$ region are shown in figure 9 along with the percentage of the exposed area of the sun's disk on February 3 . In this case the graph $\frac{N_{2}}{N_{1}}$ corresponds closely with the graph showing the percentage of the exposed area of the sun's disk. This indicates that in the $\mathrm{F}_{2}$ region at the time of this eclipse the ionization density was proportional to the first power rather than the square root of the ionizing energy of the sun.

Pedersen [15] shows that if there are $N-$ and $N+$ ions per $\mathrm{cm}^{3}$ the rate of recombination is proportional to $N^{2}$. Furthermore, if $I$ pairs of such ions are produced per $\mathrm{cm}^{3}$ each second by some 
agency, such as ultraviolet light, and $I$ is proportional to the ionizing energy of this agency, then

$$
\frac{d N}{d t}=I-\alpha N^{2}
$$

where $\alpha$ is the coefficient of recombination.

However, if the ionization is effectively of one sign, such as negative electrons surrounded chiefly by neutral gas particles, the effect of the electrons can be effectively destroyed by attachment to these neutral

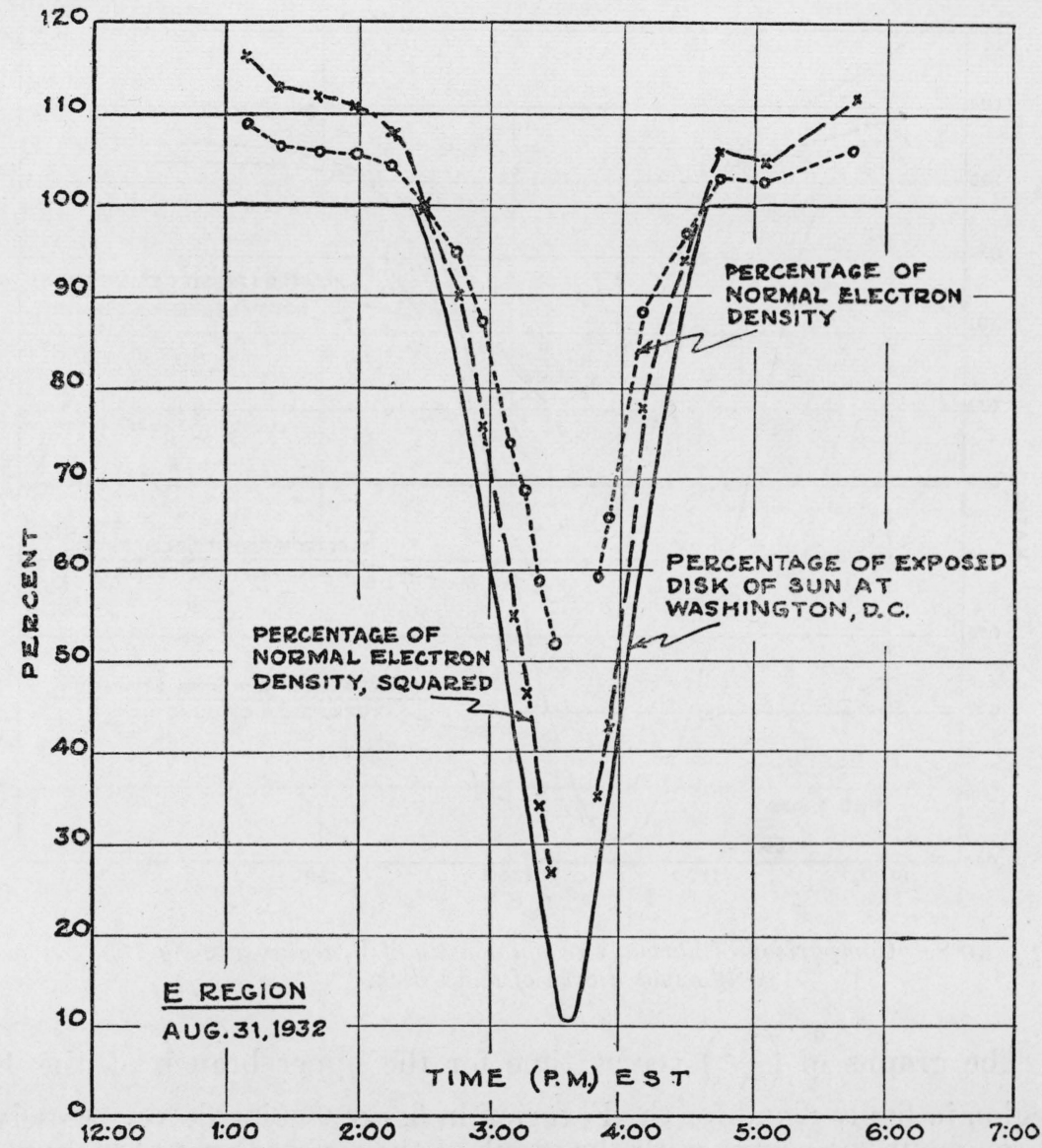

FIgURE 7.-Comparison of normal electron density of $\mathbf{F}_{1}$ region during 1932 eclipse, with exposed area of sun's disk.

gas particles. The density and temperature of the gas will be assumed constant. Then the rate at which electrons are lost by attachment is proportional to $N$ rather than $N^{2}$. An equation for this condition corresponding to equation 3 may be written as follows:

$$
\frac{d N}{d t}=I-\beta N,
$$

where $\beta$ is the coefficient of attachment.

For both equations 3 and $4 \frac{d N}{d t}=0$ at the lowest points of the graphs of $N$ versus time. In figures 6 and 9 there were very small time 
differences between the minimum of exposed area of the disk of the sun and the minimum ionization densities. Also the graphs of $\left(\frac{N_{2}}{N_{1}}\right)^{2}$ versus time for the normal $\mathrm{E}$ region in figures 6 and 7 coincide very closely throughout the eclipse with the graph of the ratio

Exposed area of sun's disk on eclipse day Total area of sun's disk.

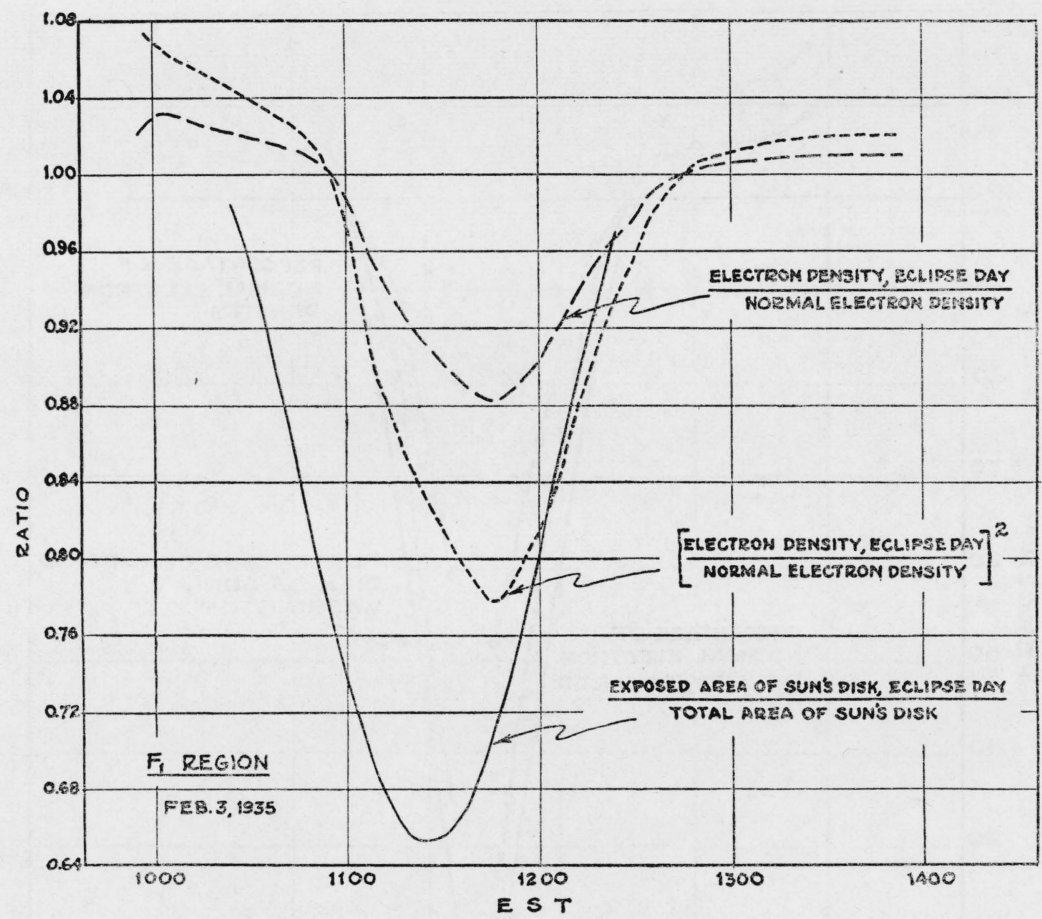

FIGURE 8.-Comparison of normal electron density of $\mathrm{F}_{1}$ region during 1935 eclipse with exposed area of sun's disk.

The graphs of $\left(\frac{N_{2}}{N_{1}}\right)$ versus time for the upper branch of the $\mathrm{E}$ region in figure 6 and for the $\mathrm{F}_{2}$ region in figure 9 coincide very closely throughout the eclipse with the graph of the exposed area of the sun's disk versus time. Therefore we may safely assume that $\frac{d N}{d t}$ was very small in comparison with either term in the right-hand members of equations 3 and 4 or for practical purposes $\frac{d N}{d t}=0$ at any point on the graphs mentioned in this paragraph for the conditions specified. This is equivalent to saying that equilibrium between ionization and recombination is reached quickly. Similar conclusions regarding the $\mathrm{F}_{1}$ region at the time of this eclipse can hardly be drawn.

It now appears that figures 6 and 7 indicate that the ionization densities of the $\mathrm{E}$ region obeyed equation 3 and that $N$ for these 
regions was proportional to the square root of the ionizing energy. These results indicate the probability that the ionization of the normal $\mathrm{E}$ region at the times of these experiments was made up of + and charges which recombined with one another when the ionizing energy

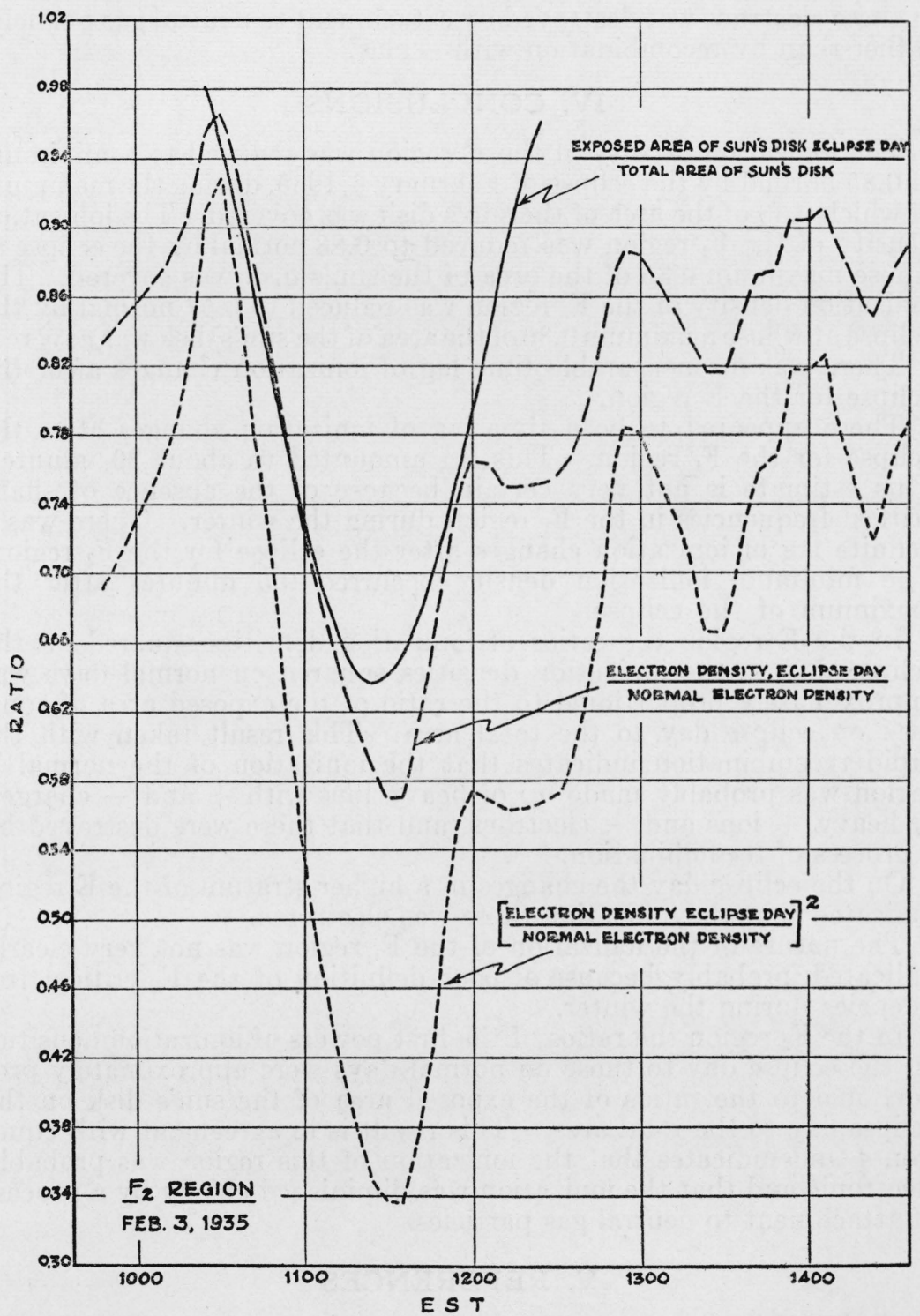

Figure 9.-Comparison of normal electron density of $\mathrm{F}_{2}$ region during 1935 eclipse with exposed area of sun's disk.

was decreased. This condition could be satisfied with heavy + and ions or with heavy + ions and - electrons.

Also figures 6 and 9 indicate that the ionization densities of the region producing the upper branch of $\mathrm{E}$ region shown in figure 2, and 
the $\mathrm{F}_{2}$ region, were proportional to the ionizing energy. These results indicate the probability that the ionization of these two regions at the times of these experiments was made up of electrons. Magneto-ionic splitting is positive evidence that the $\mathrm{F}_{2}$ region is electronic. The eclipse evidence checked this and in addition indicated that the effect of these electrons was destroyed by attachment to neutral gas particles rather than by recombination with +ions.

\section{CONCLUSIONS}

The ionization density of the $\mathrm{E}$ region was reduced to a minimum of 0.85 normal by the eclipse of February 3,1935 , during the maximum of which 0.33 of the area of the sun's disk was covered. The ionization density of the $F_{1}$ region was reduced to 0.88 normal by the eclipse at whose maximum 0.35 of the area of the sun's disk was covered. The ionization density of the $\mathrm{F}_{2}$ region was reduced to 0.57 normal by the eclipse at whose maximum 0.36 of the area of the sun's disk was covered.

There was no measurable time lag of ionization changes after the eclipse for the E region.

There appeared to be a time lag of ionization changes after the eclipse for the $F_{1}$ region. This lag amounted to about 20 minutes. This estimate is not very certain because of the absence of sharp critical frequencies in the $F_{1}$ region during the winter. There was a definite lag of ionization changes after the eclipse for the $\mathrm{F}_{2}$ region. The minimum ionization density occurred 9.5 minutes after the maximum of the eclipse.

In the $\mathrm{E}$ region the ratios of ionization densities squared on the eclipse day to the ionization densities squared on normal days was approximately proportional to the ratio of the exposed area of sun's disk on eclipse day to the total area. This result taken with the rapid recombination indicates that the ionization of the normal $\mathrm{E}$ region was probably made up of heavy ions with + and - charges, or heavy + ions and - electrons, and that these were destroyed by a process of recombination.

On the eclipse day the changes in a higher stratum of the E region indicated that the ionization there was electronic.

The nature of the ionization of the $F_{1}$ region was not very clearly indicated, probably because of poor definition of the $\mathrm{F}_{1}$ critical frequencies during the winter.

In the $\mathrm{F}_{2}$ region the ratios of the first powers of ionization densities on the eclipse day to those on normal days were approximately proportional to the ratios of the exposed area of the sun's disk on the eclipse day to the total area. This result is in agreement with equation 4 and indicates that the ionization of this region was probably electronic and that the ionization was diminished chiefly by a process of attachment to neutral gas particles.

\section{REFERENCES}

The numbers in brackets correspond to the reference numbers throughout the text.

[1] Kirby, Berkner, Gilliland, and Norton. BS J. Research 11, 289 (1933); Proc. Inst. Radio Eng. 22, 247 (Feb. 1934).

[2] J. P. Schafer and W. M. Goodall. Science 76, 444 (Nov. 11, 1932).

[3] John T. Henderson. Can. J. Research 8, 1 (Jan. 1933). 
[4] D. C. Rose. Can. J. Research 8, 15 (Jan. 1933).

[5] Kenrick and Pickard. Proc. Inst. Radio Eng. 21, 546 (April 1933).

[6] Mimno and Wang. Proc. Inst. Radio Eng. 21, 529 (April 1933).

[7] Kirby, Berkner, and Stuart. Proc. Inst. Radio Eng. 21, 757 (June 1933).

[8] Kirby, Berkner, and Stuart. BS J. Research 12, 15 (1934); Proc. Inst. Radio Eng. 22, 481 (1934).

[9] Schafer and Goodall. Nature 131, 804 (June 3, 1933).

[10] Dr. E. O. Hulburt has given a reasonable theory for these phenomena. See Hulburt, Phys. Rev. 46, 822 (Nov. 1, 1934), Terr. Mag. 40, 193 (June 1935).

[11] Gilliland. BS J. Research 11, 561 (1933); Proc. Inst. Radio Eng. 22, 236 (1934).

[12] Gilliland, Kenrick, and Norton. BS J. Research y, 1083 (1931); Proc. Inst. Radio Eng. 20, 286 (1932).

[13] Eccles. Proc. Roy. Soc. 8\%, 79 (Aug. 1912).

[14] Larmor. Phil. Mag. 48, 1025 (Dec. 1924).

[15] Pedersen. Propagation of Radio Waves. Chap. V.

Washington, December 16, 1935. 